\title{
Efficacy of single-stage breast-conserving treatment using multicatheter partial breast brachytherapy evaluated by GEC-ESTRO phase 3 trial
}

\author{
Kazuhiko Sato, MD, PhD', Hiromi Fuchikami, MD', Masahiro Kato, MD, PhD², Takahiro Shimo², Jun Kubota², \\ Naoko Takeda, MD',3, Yuko Inove, MD, PhD³, Hiroshi Seto, MD, PhD, Tomohiko Okawa, MD, PhD 5 \\ 'Department of Breast Oncology, Tokyo-West Tokushukai Hospital, Tokyo, ${ }^{2}$ Department of Radiation Oncology, Tokyo-West Tokushukai \\ Hospital, Tokyo, ${ }^{3}$ nove Ladies Clinic, Tokyo, ${ }^{4}$ Seto Hospital, Saitama, ${ }^{5}$ Preventive Medicine Center, Sano City Hospital, Tochigi, Japan
}

\begin{abstract}
Purpose: The GEC-ESTRO has reported the equivalent outcomes of partial breast irradiation (PBI) using multicatheter interstitial brachytherapy $(\mathrm{MCB})$ to whole breast irradiation (WBI) in breast-conserving therapy (BCT). We performed single-stage BCT with partial breast brachytherapy by intraoperative catheter placement. After the categorization of patients into inclusion and exclusion criteria on this trial, our databases were evaluated in order to translate it to Japanese patients.

Material and methods: Patients undergoing BCT were retrospectively examined between November 2007 and December 2015. The technique is an open-cavity implant with a dose of 32 Gy in 8 fractions. The 4-year clinical outcomes of MCB-PBI were evaluated in the 2 distinct categories, and the comparison of the outcomes of MCB-PBI with WBI was performed in patients with unfavorable features.

Results: Of a total of 501 lesions undergoing BCT, 301 lesions were treated with MCB-PBI and 200 lesions with WBI. At the median follow-up time of 52 months, the 4-year rate of ipsilateral breast tumor recurrence (IBTR)-free, disease-free (DFS), and overall survival (OS) in patients with MCB-PBI and WBI were $98.9 \%$ vs. 98.0\% $(p=0.56), 97.0 \%$ vs. $95.3 \%(p=0.78)$, and $99.6 \%$ vs. $98.2 \%(p=0.38)$, respectively. Although in exclusion cohort treated with MCB-PBI, IBTR-free, and disease-free survival were significantly worse than in inclusion cohort, non-significantly worse outcomes was demonstrated than in exclusion cohort with WBI; IBTR-free survival $(95.0 \%$ vs. $97.2 \%, p=0.24)$, and disease-free survival $(95.0 \%$ vs. $95.8 \%, p=0.31)$.

Conclusions: Single-stage BCT using MCB-PBI offered similar tumor control rates compering to WBI. However, further research is needed to define the benefit for patients with an exclusion criteria.

J Contemp Brachytherapy 2017; 9, 5: 424-430 DOI: https://doi.org/10.5114/jcb.2017.71002
\end{abstract}

Key words: breast cancer, whole breast irradiation, partial breast irradiation, interstitial brachytherapy, ipsilateral breast tumor recurrence.

\section{Purpose}

Breast-conserving therapy (BCT) has been widely accepted as an alternative treatment to mastectomy for patients with an early stage of breast cancer. An advantage of BCT is to preserve the appearance and sensation of the breast, which results in a good quality of life [1]. Recently, higher survival rate of BCT than mastectomy has been also expected [2]. On the other hand, as a disadvantage of $\mathrm{BCT}$, daily whole breast irradiation (WBI) for 3 to 5 weeks should be applied after breast-conserving surgery (BCS). A collaborate group of the meta-analysis of an early breast cancer treatment has reported that an additional irradiation to the entire breast after BCS prevent ipsilateral breast tumor recurrence (IBTR), which is also related to increased rate of survival [3]. Even if the benefit of adjuvant radiotherapy was insignificant in elderly patients with low-risk tumor, no subsets of patients in whom radiation therapy could be safely avoided have not been reported [4]. In the United States, however, more than $20 \%$ of patients undergoing BCS have not receive an adjuvant radiotherapy because of long-lasting of treatment [5].

To reduce the burden of WBI, partial breast irradiation (PBI) techniques has been introduced to deliver radiation to limited field of the breast. PBI using multicatheter interstitial brachytherapy (MCB) has been initially reported, and this is the most matured data including relatively small randomized study [6]. Recently, the
Address for correspondence: Kazuhiko Sato, MD, PhD, Department of Breast Oncology, Tokyo-West Tokushukai Hospital, 3-1-1 Matsubara, Akishima, Tokyo 196-0003, Japan, phone: +81 42-500-4433, fax: +81 42-500-4432, e-mail: kazsato.boc@gmail.com
Received: 08.06 .2017

Accepted: 10.10 .2017

Published: 30.10 .2017 
Groupe Européen de Curiethérapie - European Society for Therapeutic Radiology and Oncology (GEC-ESTRO) has reported the outcomes of a large randomized non-inferiority trial comparing the efficacy of MCB-PBI with WBI with a tumor-bed boost after BCS [7]. At a median follow-up of 6.6 years, the cumulative incidence of local recurrence was $1.44 \%$ with MCB-PBI and $0.92 \%$ with WBI, of these $0.52 \%$ difference $(p=0.42)$ was below the non-inferiority margin. Moreover, late toxicities and cosmetic results have been shown to be similar between two radiotherapies [8]. Based on this trial, MCB-PBI could be considered as an alternative method to WBI after BCS for low-risk breast cancer patients.

However, it would be difficult to translate the evidence directly into Japanese community practice because of different clinical environments and patient profiles. In our institution, most of the patients are from small and rural communities with difficult access to radiotherapy. MCB-PBI had to be chosen as an alternative treatment option to WBI for many patients. Although our indication for MCB-PBI were basically the same as those in the GEC-ESTRO protocol, catheters were placed at the time of primary surgery. Therefore, strict selection criteria could not be achieved because the final pathology report was not available. In this study, patients could be categorized into two cohort by using factors relating to inclusion and exclusion criteria on the GEC-ESTRO trial, and our database of patients with two distinct cohort were evaluated.

\section{Material and methods}

\section{Patient and surgery}

In the retrospective cohort study, consecutive patients who underwent BCS followed by an adjuvant radiotherapy were examined between November 2007 and December 2015. BCS has been applied for patients with Tis-2 $(\leq 3 \mathrm{~cm})$ N0-1 breast cancer to attempt a negative surgical margin of at least $1 \mathrm{~cm}$, and to achieve an acceptable cosmetic outcome. By an evaluation of margin using specimen-mammography during surgery indicating extension of the tumor near the margin, additional tissue was resected. An overlying skin was also excised to maintain sufficient skin-surface distance for superficial tumor. If sentinel nodes were revealed positive for metastasis during surgery or positive axillary nodes were found by pre-operative fine-needle biopsy, a complete axillary dissection had been performed. From October 2008, after obtaining approval from the institutional review board, MCB-PBI has also been performed. The inclusion criteria of our MCB-PBI treatment had been similar to those of the GEC-ESTRO trial; $\geq 40$ years old, tumor diameter $\leq 3 \mathrm{~cm}, \mathrm{pN} 0-1 \mathrm{mi}$, and negative surgical margin. The retrospective chart reviews were performed for clinic-pathological features including age, tumor size, nuclear grade, lymph node status, surgical margins, estrogen receptor (ER), and human epidermal growth factor receptor 2 (HER2) status. In this study, in order to simplify and generalize the criteria, no tumor cells at the resection surface was defined as negative margin introduced by American Society for Radiation Oncology and Society of Surgical
Oncology [9]. Also, lymphovascular status was not included for the criteria because the extent was not specifically reported, which were different from the inclusion criteria of the GEC-ESTRO trial. There were no patients referred for BRCA $1 / 2$ testing.

\section{Radiation techniques}

Patients undergoing BCT have received one out of two types of adjuvant radiation techniques, MCB-PBI or WBI. MCB-PBI was performed just after BCS, and WBI was applied after an adjuvant chemotherapy when needed. In patients receiving MCB-PBI, the basic technique is an open-cavity implant, which has been introduced elsewhere [10]. Before surgery, a contrast enhanced computed tomography (CT) was obtained to decide implant geometry using the Nucletron PLATO treatment planning system (Version UPS 11.3; Nucletron Trading BV, Veenendaal, The Netherlands). Just after removal of the tumor, surgical clips were placed to mark the extent of the cavity. The insertion of catheters was performed under direct visualization of the tumor cavity, intraoperative open-cavity implant technique. The stainless steel rigid needles were inserted under a template guide with pre-operative CT simulation and flexible plastic catheters for the introduction of iridium wires replaced from the needles. After surgery, a non-contrast CT identifying the cavity with the seroma and surgical clips was acquired to provide dose-volume histograms. The planning target volume included the surgical cavity delineated by ligating clips, as well as a $10-20 \mathrm{~mm}$ margin with the maximum dose to the skin and chest at $<75 \%$ of the prescribed dose. High-dose-rate interstitial brachytherapy with ${ }^{192} \mathrm{Ir}$ was performed in an accelerated manner with a dose of 32 Gy in eight fractions over 5-6 days. Each radiotherapy was delivered daily with 6 hours or longer between the fractions. For the other patients, WBI was performed with a total dose of $50 \mathrm{~Gy}$ in fractions of $2 \mathrm{~Gy}$, using a threedimensional treatment planning system (Philips' Pinnacle 3 treatment planning system; Fitchburg, WI, USA). The radiation field was depended on the risk of local recurrence; patients with risk factors such as positive margins and young age ( $<40$ years) generally received a subsequent $10 \mathrm{~Gy}$ boost to the tumor bed using electrons; regional nodal irradiation was added in patients with $\geq 4$ positive nodes.

\section{Follow-up and data analysis}

Post-treatment follow-up included clinical examination at every 3-4 months and an annual mammography. Contrast-enhanced breast magnetic resonance imaging was also performed every year for the first 5 years. If any lesion of suspected recurrence was detected, a needle biopsy was performed to confirm IBTR and regional nodal failure. IBTR was classified as "local recurrence", but it was also classified into "tumor bed recurrence" and "treatment failure elsewhere" on the basis of the tumor location in relation to the lumpectomy cavity [11]. Tumor bed recurrence was considered as a true recurrence located within or immediately adjacent to the lumpec- 
tomy cavity. Treatment failure elsewhere was generally regarded as a new primary cancer located at a distance of several centimeters away from the cavity. The axillary, infraclavicular, or supraclavicular lymph node recurrence was classified as "regional recurrence".

The 4-year clinical outcomes of MCB-PBI were compared with WBI in the entire cohort in respect to the rate of IBTR-free survival (IBTR-FS), disease-free survival (DFS), and overall survival (OS). The outcomes of MCBPBI were also evaluated in the distinct categories between the inclusion and exclusion criteria, to compare them to those of the GEC-ESTRO trial. Next, in order to evaluate a possibility to extend the candidate of MCB-PBI for patients with unfavorable risk factors for disease recurrences, clinically relevant factors in the GEC-ESTRO criteria have been analyzed whether the type of radiation technique was independently selected or not, and the comparison of the outcomes of patients treated by MCB-PBI with WBI was performed in patients excluded from the GEC-ESTRO trial.

\section{Statistical methods}

The $\chi^{2}$ test was used to analyze associations between categorical variables. Student's unpaired $t$-test was used to analyze differences between continuous variables. Survival curves were calculated using the Kaplan-Meier method and statistically compared using the log-rank test. The Cox proportional hazards model was used for univariate and multivariate analysis to determine significant risk factors for IBTR and locoregional and distant recurrences. Hazard ratios (HRs) and 95\% confidence intervals were calculated for each covariate. Probability $(p)$ values of $<0.05$ were considered to indicate statistical significance. All time intervals were calculated from the date of BCS to the date of first recurrence or of last patients' visit. Bilateral breast cancers treated by BCT were counted as two different patients in the analysis.

\section{Results}

\section{Characteristics and outcomes of patients undergoing breast-conserving therapy}

Of a total of 501 lesions in 493 patients undergoing BCT, 301 lesions in 297 patients were treated with MCBPBI, and 200 lesions in 196 patients with WBI, of which number of lesions were calculated as a distinct patient hereafter. Patient characteristics are shown in Table 1. The median follow-up time exceeded 48 months in both treatment groups with nonsignificant longer trend for patients with WBI than with MCB-PBI (50 vs. 54 months, $p=0.06)$. There were significant differences in patients' age, nodal involvements, Tis, and positive margin status observed in patients with MCB-PBI than with WBI. Two patients $(0.7 \%)$ with MCB-PBI received an additional WBI due to their unfavorable final pathology, and 54 patients $(27 \%)$ with WBI received a boost radiotherapy. There were no differences in the receipt of adjuvant systemic therapies between two groups. In patients undergoing BCT using MCB-PBI or WBI, a total of 24 (4.8\%) patients developed disease recurrence or non-breast can- cer-related death at their first event. As the first breast cancer failure in patients receiving MCB-PBI and WBI, IBTRs, regional recurrences, contralateral breast cancer, and distant recurrences were found in $5(1.7 \%, 95 \%$ CI: $0.22-3.10), 3(1.0 \%, 95 \%$ CI: $0-2.11), 2$ (0.7\%, 95\% CI: $0-1.58), 6(2.0 \%, 95 \% \mathrm{CI}: 0.41-3.57)$, and $5(2.5 \%, 95 \% \mathrm{CI}$ : $0.34-4.66), 0,0,5$ (2.5\%, 95\% CI: 0.34-4.66) patients, respectively. There were $2(1 \%)$ breast cancer-related fatalities observed only in patients with WBI. Two patients $(0.7 \%)$ with WBI-PBI and one patients $(0.5 \%)$ with WBI passed away due to the other reason. Thus, the 4-year rate of IBTR-FS, DFS, and OS in patients with MCB-PBI and WBI were $98.9 \%$ vs. $98.0 \%(p=0.56$; Figure $1 \mathrm{~A}), 97.0 \%$ vs. $95.3 \%(p=0.78$; Figure $1 \mathrm{~B})$, and $99.6 \%$ vs. $98.2 \%$ $(p=0.38$; Figure $1 C)$, respectively. There were nonsignificant differences in any survival curves according to the radiation groups.

\section{Impact of eligibility criteria on the outcomes of multicatheter interstitial brachytherapy - partial breast irradiation}

To evaluate the impact eligibility criteria on the outcomes of 301 patients with MCB-PBI, the treatment results between two categories were analyzed. A total of 237 patients $(78.7 \%)$ met the inclusion criteria of GEC-ESTRO trial, and $64(21.3 \%)$ did not (Table 2). Among 8 locoregional recurrences in patients receiving MCB-PBI, there were 5 IBTRs in exclusion and 3 regional recurrences in inclusion criteria cohort. Of these IBTRs, 3 recurrences were observed in the "tumor bed" and 2 were "elsewhere". There were 6 distant metastases observed, including 2 patients in inclusion cohort and 4 in exclusion cohort. There were 2 contralateral breast cancer and 2 non-breast cancer-related deaths observed. Therefore, Kaplan-Meier estimates for the 4-year IBTR-FS in patients with inclusion and exclusion cohort were 100\% and $95.0 \%(p<0.0001)$, and DFS were $97.6 \%$ and $95.0 \%$ $(p<0.05)$, respectively. The latter cohort demonstrated a significantly higher rate of locoregional and distant recurrences.

\section{Impact of exclusion criteria on outcomes between multicatheter interstitial brachytherapy - partial breast irradiation and whole breast irradiation}

Predictive factors of unfavorable prognosis related to enrollment criteria of the GEC-ESTRO trial were analyzed in patients undergoing MCB-PBI and WBI after BCS. There were only 4 patients with larger than $3 \mathrm{~cm}$ tumor diameter, and no breast cancer-related death was observed. Therefore, the factors including age, lymph node involvement, margin status, and radiation technique were evaluated for the risk of IBTR, and any breast cancer-related events. On univariate and multivariate analysis, lymph node metastasis and positive surgical margin status were selected as a predictor of the both events. Types of radiation technique were not associated with increased IBTR (HR=0.69; 95\% CI: 0.20-2.39; $p=0.56$ ), nor any breast cancer event $(\mathrm{HR}=0.89 ; 95 \% \mathrm{CI}$ : 0.39-2.03; $p=0.78$ ) (Table 3). 
Table 1. Patients' undergoing breast-conserving therapy characteristics

\begin{tabular}{|c|c|c|c|c|}
\hline \multirow[t]{2}{*}{ Characteristic } & \multirow[t]{2}{*}{ All patients with BCT $(n=501)$} & \multicolumn{3}{|c|}{ Radiation technique } \\
\hline & & $\begin{array}{l}\text { MCB-PBI } \\
(n=301) \\
\end{array}$ & $\begin{array}{c}\text { WBI } \\
(n=200)\end{array}$ & $p$ \\
\hline Median follow-up (IQR), months & $52(24.0-77.0)$ & $50(24.0-76.0)$ & $54(25.5-83.0)$ & 0.06 \\
\hline Average age (IQR), years & $55.0(46-64)$ & $52.7(44-61)$ & $56.5(44-61)$ & $<0.001$ \\
\hline \multicolumn{5}{|l|}{ pT stage* (\%) } \\
\hline pTis & $59(11.8)$ & $28(9.3)$ & $31(15.5)$ & \multirow{4}{*}{0.03} \\
\hline pT1 & $398(79.4)$ & $250(83.1)$ & $148(74)$ & \\
\hline pT2 & $42(8.4)$ & $21(7.0)$ & $21(10.5)$ & \\
\hline Unknown & $2(0.4)$ & $2(0.6)$ & 0 & \\
\hline \multicolumn{5}{|l|}{ Nuclear grade (\%) } \\
\hline Grade 1 & $355(70.8)$ & $226(75.1)$ & $129(64.5)$ & \multirow{3}{*}{0.34} \\
\hline Grade 2-3 & $81(16.2)$ & $47(15.6)$ & $34(17)$ & \\
\hline Unknown & $65(13.0)$ & $28(9.3)$ & $37(18.5)$ & \\
\hline \multicolumn{5}{|l|}{ Positive lymph node (\%) } \\
\hline 0 & $396(79.0)$ & $271(90.0)$ & $125(62.5)$ & \multirow{5}{*}{$<0.0001$} \\
\hline Micro-metastasis & $9(1.8)$ & $5(1.7)$ & $4(2)$ & \\
\hline $1-3$ & $77(15.4)$ & $19(6.3)$ & $58(29)$ & \\
\hline$\geq 4$ & $17(3.4)$ & $6(2.0)$ & $11(5.5)$ & \\
\hline No assessment & $2(0.4)$ & 0 & $2(1)$ & \\
\hline \multicolumn{5}{|l|}{ ER status (\%) } \\
\hline Negative & $69(13.8)$ & $42(14.0)$ & $27(13.5)$ & \multirow{2}{*}{0.89} \\
\hline Positive & $432(86.2)$ & $259(86.0)$ & $173(86.5)$ & \\
\hline \multicolumn{5}{|l|}{ HER2 status (\%) } \\
\hline Negative & $445(88.8)$ & $264(87.7)$ & $181(90.5)$ & \multirow{2}{*}{0.33} \\
\hline Positive & $56(11.2)$ & $37(12.3)$ & $19(9.5)$ & \\
\hline \multicolumn{5}{|l|}{ Surgical margin (\%) } \\
\hline Negative & $427(85.2)$ & $269(89.4)$ & $159(79.5)$ & \multirow{2}{*}{$<0.005$} \\
\hline Positive & $74(14.8)$ & $32(10.6)$ & $41(20.5)$ & \\
\hline \multicolumn{5}{|l|}{ Additional radiotherapy (\%) } \\
\hline WBI & $2(0.4)$ & $2(0.7)$ & - & \\
\hline Boost & $54(10.8)$ & - & $54(27)$ & \\
\hline \multicolumn{5}{|l|}{ Hormone therapy (\%) } \\
\hline Yes & $420(83.8)$ & $250(83.1)$ & $170(85)$ & \multirow{2}{*}{0.56} \\
\hline No & $81(16.2)$ & $51(16.9)$ & $30(15)$ & \\
\hline \multicolumn{5}{|l|}{ Chemotherapy (\%) } \\
\hline Yes & $182(36.3)$ & $102(33.9)$ & $80(40)$ & \multirow{2}{*}{0.16} \\
\hline No & 319 (63.7) & 199 (66.1) & $120(60)$ & \\
\hline
\end{tabular}

$B C T$ - breast-conserving therapy, $M C B-P B I$ - multicatheter interstitial brachytherapy - partial breast irradiation, WBI - whole breast irradiation 
A

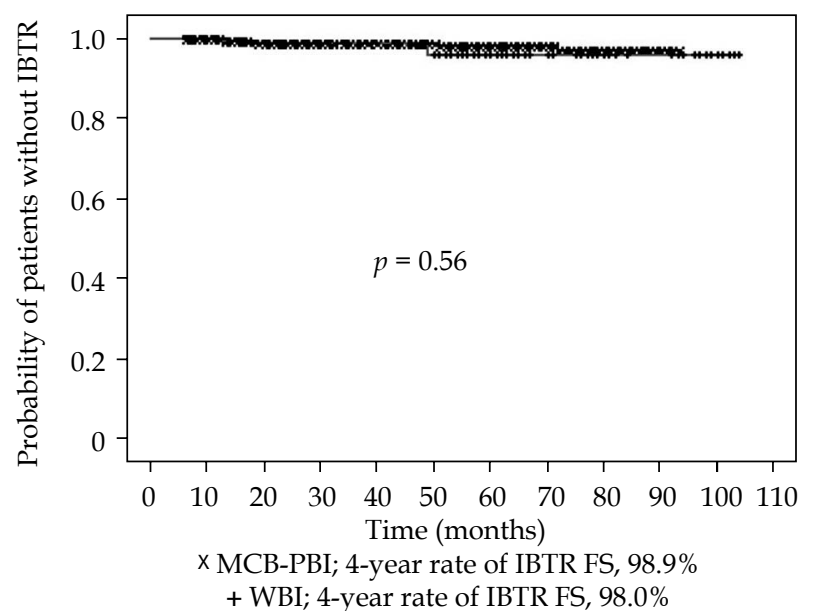

C

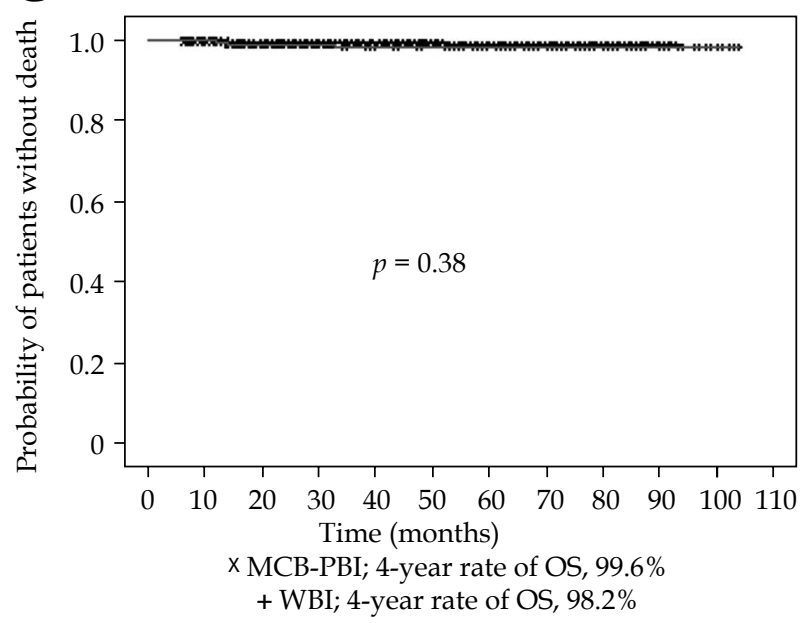

B

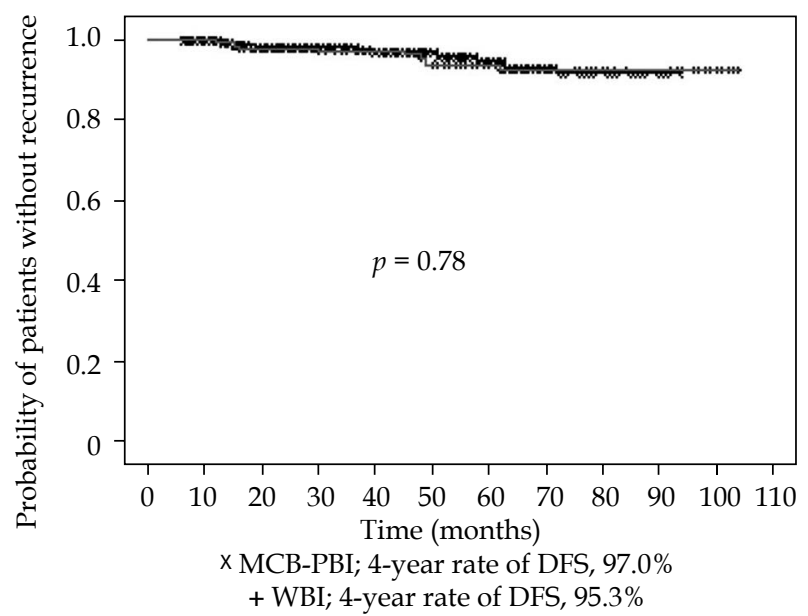

Fig. 1. 4-year rate of ipsilateral breast tumor recurrencefree survival (IBTR-FS), disease-free survival (DFS), and overall survival (OS) in patients with multicatheter interstitial brachytherapy - partial breast irradiation (MCB-PBI) and whole breast irradiation (WBI)

Table 2. Characteristics related to eligibility in patients undergoing multicatheter interstitial brachytherapy partial breast irradiation and whole breast irradiation

\begin{tabular}{|c|c|c|c|c|c|c|}
\hline \multirow[t]{2}{*}{ Characteristic } & \multicolumn{3}{|c|}{ Inclusion criteria $(n=325)$} & \multicolumn{3}{|c|}{ Exclusion criteria $(n=176)$} \\
\hline & $\begin{array}{l}\text { MCB-PBI } \\
(n=237)\end{array}$ & $\begin{array}{c}\text { WBI } \\
(n=88)\end{array}$ & $p$ & $\begin{array}{l}\text { MCB-PBI } \\
(n=64)\end{array}$ & $\begin{array}{c}\text { WBI } \\
(n=112)\end{array}$ & $p$ \\
\hline \multicolumn{7}{|l|}{ Age (\%) } \\
\hline$<40$ years & 0 & 0 & - & $12(18.8)$ & $25(22.3)$ & 0.58 \\
\hline$\geq 40$ years & $237(100)$ & $88(100)$ & & $52(81.2)$ & $87(77.7)$ & \\
\hline \multicolumn{7}{|c|}{ Tumor diameter (\%) } \\
\hline$\leq 3.0 \mathrm{~cm}$ & $237(100)$ & $88(100)$ & - & $62(96.9)$ & $110(98.2)$ & 0.57 \\
\hline$>3.0 \mathrm{~cm}$ & 0 & 0 & & $2(3.1)$ & $2(1.8)$ & \\
\hline \multicolumn{7}{|l|}{ Nodal status (\%) } \\
\hline pNO or pN1 mi & $237(100)$ & $88(100)$ & - & $39(60.9)$ & $43(38.4)$ & $<0.005$ \\
\hline pN1 & 0 & 0 & & 25 (39.1) & 69 (61.6) & \\
\hline \multicolumn{7}{|c|}{ Surgical margin (\%) } \\
\hline Negative & 0 & 0 & - & $32(50)$ & $71(63.4)$ & 0.083 \\
\hline Positive & 237 (100) & 88 (100) & & $32(50)$ & $41(36.6)$ & \\
\hline
\end{tabular}

$M C B-P B I$ - multicatheter interstitial brachytherapy - partial breast irradiation, WBI-whole breast irradiation 
Table 3. Univariate and multivariate analysis of prognostic factors for ipsilateral breast tumor recurrence and breast cancer event

\begin{tabular}{|c|c|c|c|c|c|c|}
\hline \multirow[t]{3}{*}{ Variables } & \multicolumn{3}{|c|}{ IBTR } & \multicolumn{3}{|c|}{ Locoregional and distant recurrences } \\
\hline & \multicolumn{2}{|c|}{$p$-value } & \multirow[t]{2}{*}{$\mathrm{HR}(95 \% \mathrm{Cl})$} & \multicolumn{2}{|c|}{$p$-value } & \multirow[t]{2}{*}{$\mathrm{HR}(95 \% \mathrm{Cl})$} \\
\hline & Univariate & Multivariate & & Univariate & Multivariate & \\
\hline Age: $\geq 40$ vs. $<40$ years & 0.25 & - & $2.49(0.53-11.72)$ & 0.89 & - & $0.90(0.21-3.85)$ \\
\hline $\begin{array}{l}\text { Axillary node: } \\
\text { negative vs. positive }\end{array}$ & $<0.05$ & $<0.05$ & $4.09(1.18-14.12)$ & $<0.05$ & $<0.05$ & $2.75(1.19-6.36)$ \\
\hline $\begin{array}{l}\text { Margin status: } \\
\text { negative vs. positive }\end{array}$ & $<0.01$ & $<0.01$ & $5.58(1.54-20.29)$ & 0.075 & 0.068 & $2.55(0.93-6.97)$ \\
\hline RT: WBI vs. MCB-PBI & 0.56 & - & $0.69(0.20-2.39)$ & 0.78 & - & $0.89(0.39-2.03)$ \\
\hline
\end{tabular}

IBTR - ipsilateral breast tumor recurrence, RT - radiotherapy, MCB-PBI - multicatheter interstitial brachytherapy - partial breast irradiation, WBI - whole breast irradiation

In patients undergoing WBI, 88 patients $(44 \%)$ were classified into the inclusion criteria, and $112(56 \%)$ in the exclusion criteria. There was significantly more patients with lymph node involvement in WBI group among exclusion cohort than in MCB-PBI $(p<0.005)$ (Table 2). As per our expectation, the 4-year probability of IBTR-FS and DFS in an exclusion patients was significantly worse than in an inclusion cohort for both of treatment groups. However, no significant difference in the outcomes between two radiotherapies was demonstrated in the respect to either IBTR-FS (95.0\% vs. $97.2 \%, p=0.24)$, DFS $(95.0 \%$ vs. $95.8 \%, p=0.31)$, and OS $(100 \%$ vs. $99.0 \%$, $p=0.80)$ in patients with exclusion criteria.

\section{Discussion}

The incidence rate of breast cancer has been much lower in Japan than in Europe and North America, but has significantly increased at the early stage, and the rate of BCT has been applied to more than $60 \%$ of patients [12]. However, among them, up to $20 \%$ do not receive adjuvant radiation therapy like in the United States [13]. PBI with less treatment burden has a great benefit if the efficacy of local control. However, few reports evaluated the outcomes of PBI after BCS in Japanese breast cancer patients. In this study, we have shown an excellent clinical outcomes of MCB-PBI with outcomes of $1.7 \%$ IBTR rate in the entire cohort that were quite similar to those in the GEC-ESTRO trial, where the rate of IBTR was 1.4\% [7]. According to the Pooled Registry of Multicatheter Interstitial Sites (PROMIS) study, showing long-term follow-up outcomes from more than 1,000 patients with a variety of background, the 5- and 10-years local recurrence rate was $3.8 \%$ and $7.6 \%$, respectively [14]. Those evidences could support MCB-PBI introduction into Japanese practice.

Our technique of BCS was an open-cavity surgery and the catheters were placed at the time of BCS, intraoperative open-cavity implant. The greatest advantage of this single-stage BCT was the same as an intraoperative radiation therapy (IORT), which do not need an additional invasive procedure. However, the IORT technique can cover only the spherical dose distribution around the cavity.
In fact, according to two large randomized clinical trials, $50 \mathrm{kv}$ photons in the targeted intraoperative radiotherapy (TARGIT-A) trial [15] and large electrons in the electron intraoperative therapy (ELIOT) trial [16], the rate of IBTR has been observed almost 3 times higher than that of WBI in both trials. Although these techniques including ours should start radiotherapy without final pathology, manual replacement of the catheters or applicator achieved an accurate delineation of a clinical target volume because the tumor cavity was directly visualized. In the TARGIT-A trial, low recurrence rates were obtained in patients receiving IORT immediately after lumpectomy, even though the post-pathology stratum were highly selected for favorable pathological criteria.

In this study, patients with an inclusion criteria of the GEC-ESTRO trial, though small and short follow-up period, showed excellent 4-year IBTR-free rates of $100 \%$, with four important factors (age, tumor size, nodal status, and margin) selected. Node-positive patients could undertake WBI with axillary radiation instead of axillary dissection [17]. Extension of the radiation field to the axilla and subclavicular area should be still under a debate [18]. Although with a sufficient systemic treatment, there were not significantly differences in local control between two radiation groups, and regional nodes metastases were found 3 patients only in MCB-PBI. The addition of regional node irradiation to WBI and axillary node irradiation should be consider for node-positive patients. On our multivariate analysis, positive margin status has been selected as the independent risk factor associated with IBTR in patients not only receiving MCB-PBI, but also WBI. In our technique of MCB-PBI, interstitial implants was placed intraoperatively with an open surgical cavity, and PBI should be performed without final pathology. In the experimental arm of the TARGIT-A trial [19], where additional WBI was prescribed in patients with risk factors in terms of size, histology, margin status, node positivity, etc., at a median follow-up of 18.0 months in 232 patients, only one IBTR was observed resulting a local relapse-free survival to be $99.1 \%$, which was below the prospectively defined stopping rules [20]. In order to achieve more effective local control by MCB-PBI, the TARGIT-E trial will give us promising strategy. 
In this study, we reviewed our single-institution experience of BCT using MCB-PBI and WBI in Japanese patients with the inclusion and exclusion criteria of the GEC-ESTRO trial. Two hypotheses have been raised to support such an excellent results. MCB-PBI has an equal or more tumor control effect, and the additional benefit of WBI in local recurrence has been diminished by effective systemic therapy. Recent advances in adjuvant systemic therapy have reduced the risk of distant metastases and local recurrences in BCT as well, which contributes to less clear margin in BCS [9] and the omission of axillary clearance for patients with positive sentinel node metastasis [17]. To our knowledge, this study includes the largest cohort of Asian patients undergoing MCB-PBI. Single-stage BCT using MCB-PBI offered similar tumor control rates compering to WBI. Although there was no negative impact of MCB-PBI on the outcomes in patients even with an exclusion criteria, further research is needed to define optimal candidates based on molecular subtype and genomic profiling [21].

\section{Disclosure}

Authors report no conflict of interest.

\section{References}

1. Han J, Grothuesmann D, Neises M et al. Quality of life and satisfaction after breast cancer operation. Arch Gynecol Obstet 2010; 282: 75-82.

2. Agarwal S, Pappas L, Neumayer L et al. Effect of breast conservation therapy vs. mastectomy on disease-specific survival for early-stage breast cancer. JAMA Surg 2014; 149: 267-274.

3. Early Breast Cancer Trialists' Collaborative Group (EBCTCG), Darby S, McGale P, et al. Effect of radiotherapy after breastconserving surgery on 10-year recurrence and 15-year breast cancer death: meta-analysis of individual patient data for 10,801 women in 17 randomised trials. Lancet 2011; 378: 1707-1716.

4. Giordano SH. Radiotherapy in older women with low-risk breast cancer: why did practice not change? J Clin Oncol 2012; 30: 1577-1578.

5. Churilla TM, Donnelly PE, Leatherman ER et al. Total Mastectomy or Breast Conservation Therapy? How Radiation Oncologist Accessibility Determines Treatment Choice and Quality: A SEER Data-base Analysis. Breast J 2015; 21: 473-480.

6. Polgár C, Fodor J, Major $T$ et al. Breast-conserving therapy with partial or whole breast irradiation: ten-year results of the Budapest randomized trial. Radiother Oncol 2013; 108: 197-202.

7. Strnad V, Ott OJ, Hildebrandt G et al. 5-year results of accelerated partial breast irradiation using sole interstitial multicatheter brachytherapy versus whole-breast irradiation with boost after breast-conserving surgery for low-risk invasive and in-situ carcinoma of the female breast: a randomised, phase 3, non-inferiority trial. Lancet 2016; 387: 229-238.

8. Polgár C, Ott OJ, Hildebrandt G et al. Late side-effects and cosmetic results of accelerated partial breast irradiation with interstitial brachytherapy versus whole-breast irradiation after breast-conserving surgery for low-risk invasive and in-situ carcinoma of the female breast: 5 -year results of a randomised, controlled, phase 3 trial. Lancet Oncol 2017; 18: 259-268.

9. Buchholz TA, Somerfield MR, Griggs JJ et al. Margins for breast-conserving surgery with whole-breast irradiation in stage I and II invasive breast cancer: American Society of Clinical Oncology endorsement of the Society of Surgical
Oncology/ American Society for Radiation Oncology consensus guideline. J Clin Oncol 2014; 32: 1502-1506.

10. Sato K, Mizuno $Y$, Kato $M$ et al. Intraoperative Open-Cavity Implant for Accelerated Partial Breast Irradiation Using High-Dose Rate Multicatheter Brachytherapy in Japanese Breast Cancer Patients: A Single-Institution Registry Study. JCT 2012; 3: 822-830.

11. Yi M, Buchholz TA, Meric-Bernstam F et al. Classification of ipsilateral breast tumor recurrences after breast conservation therapy can predict patient prognosis and facilitate treatment planning. Ann Surg 2011; 253: 572-579.

12. Kurebayashi J, Miyoshi Y, Ishikawa T et al. Clinicopathological characteristics of breast cancer and trends in the management of breast cancer patients in Japan: Based on the Breast Cancer Registry of the Japanese Breast Cancer Society between 2004 and 2011. Breast Cancer 2015; 22: 235-244.

13. Saji S, Hiraoka M, Tokuda Y et al. Trends in local therapy application for early breast cancer patients in the Japanese Breast Cancer Society Breast Cancer Registry during 20042009. Breast Cancer 2012; 19: 1-3.

14. Kamrava M, Kuske RR, Anderson B et al. Outcomes of Breast Cancer Patients Treated with Accelerated Partial Breast Irradiation Via Multicatheter Interstitial Brachytherapy: The Pooled Registry of Multicatheter Interstitial Sites (PROMIS) Experience. Ann Surg Oncol 2015; 22 Suppl 3: S404-411.

15. Vaidya JS, Wenz F, Bulsara M et al. Risk-adapted targeted intraoperative radiotherapy versus whole-breast radiotherapy for breast cancer: 5-year results for local control and overall survival from the TARGIT-A randomised trial. Lancet 2014; 383: 603-613.

16. Veronesi U, Orecchia R, Maisonneuve P et al. Intraoperative radiotherapy versus external radiotherapy for early breast cancer (ELIOT): a randomised controlled equivalence trial. Lancet Oncol 2013; 14: 1269-1277.

17. Giuliano AE, Hunt KK, Ballman KV et al. Axillary dissection vs no axillary dissection in women with invasive breast cancer and sentinel node metastasis: a randomized clinical trial. JAMA 2011; 305: 569-575.

18. Whelan TJ, Olivotto IA, Parulekar WR et al. Regional Nodal Irradiation in Early-Stage Breast Cancer. N Engl J Med 2015; 373: 307-316.

19. Neumaier C, Elena S, Grit W et al. TARGIT-E(lderly) prospective phase II study of intraoperative radiotherapy (IORT) in elderly patients with small breast cancer. BMC Cancer 2012; 12: 171.

20. Wenz FK, The German TARGIT E Trialists. TARGIT E(lderly): Prospective phase II study of intraoperative radiotherapy (IORT) in elderly patients with small breast cancer. J Clin Oncol 2016; 34 (suppl; abstr 1044).

21. Drukker CA, Elias SG, Nijenhuis MV et al. Gene expression profiling to predict the risk of locoregional recurrence in breast cancer: a pooled analysis. Breast Cancer Res Treat 2014; 148: 599-613. 